\title{
La enseñanza virtual y la deserción escolar en la pandemia 2019
}

Virtual education and school desertion in the 2019 pandemic

1 Pablo Enrique Hernández Domínguez

https://orcid.org/0000-0003-3862-1489.

Universidad Técnica de Ambato, Facultad de Ciencias Humanas y de la Educación,

Carrera de Educación Básica. Ambato, Ecuador

pe.hernandez@uta.edu.ec

2 Edgar Bladimir Sánchez Vaca (iD) https://orcid.org/0000-0003-1002-405X

Universidad Técnica de Ambato, Facultad de Ciencias Humanas y de la Educación,

Carrera de Educación Básica. Ambato, Ecuador

eb.sanchez@uta.edu.ec

3 María José Alarcón Chávez (iD) https://orcid.org/0000-0002-0535-4225.

Universidad Técnica de Ambato, Facultad de Ciencias Humanas y de la Educación,

Carrera de Educación Básica. Ambato, Ecuador

malarcon4354@uta.edu.ec

Artículo de Investigación Científica y Tecnológica

Enviado: 24/12/2021

Revisado: 29/12/2021

Aceptado: $12 / 01 / 2022$

Publicado:08/03/2023

DOI: https://doi.org/10.33262/concienciadigital.v6i1.4.2046

Cítese:

Hernández Domínguez, P. E., Sánchez Vaca, E. B., \& Alarcón Chávez, M. J. (2023). La enseñanza virtual y la deserción escolar en la pandemia 2019. ConcienciaDigital, 6(1.4), 995-1012. https://doi.org/10.33262/concienciadigital.v6i1.4.2046

CONCIENCIA DIGITAL, es una Revista Multidisciplinar, Trimestral, que se publicará en soporte electrónico tiene como misión contribuir a la formación de profesionales competentes con visión humanística y crítica que sean capaces de exponer sus resultados investigativos y científicos en la misma medida que se promueva mediante su intervención cambios positivos en la sociedad. https://concienciadigital.org

La revista es editada por la Editorial Ciencia Digital (Editorial de prestigio registrada en la Cámara Ecuatoriana de Libro con No de Afiliación 663) www.celibro.org.ec 


\section{Palabras} claves:

Enseñanza, virtual, deserción, pandemia, aprendizaje.

Keywords: teaching, virtual, desertion, pandemic, learning.
Resumen

Introducción: La presente investigación tuvo la finalidad de analizar la influencia de la enseñanza virtual en la deserción escolar de los estudiantes de Educación General Básica de la Unidad Educativa "Jerusalén" del Cantón Ambato. Objetivo. Analizar la influencia de la enseñanza virtual en la deserción escolar de los estudiantes de Educación General Básica de la Unidad Educativa "Jerusalén" del Cantón Ambato. Metodología. La metodología utilizada tuvo un enfoque cuali-cuantitativo, se centró en un análisis de alcance exploratorio y descriptivo en la población seleccionada. Como técnica para la recolección de la información se utilizó la encuesta validada con un cuestionario de diez preguntas objetivas de opción múltiple con una población de 116 estudiantes. Después de la recopilación de datos, se probó la hipótesis planteada. Resultado. Los resultados obtenidos demuestran que un gran porcentaje de estudiantes abandonan los estudios por tener escasas herramientas tecnológicas. Esto ha provocado sentimientos de frustración, en estudiantes, docentes y padres de familia, dificultando también el proceso de enseñanza aprendizaje. Conclusiones. Como conclusión se puede determinar que de un total de 116 estudiantes investigados tan solo 54 estudiantes poseen una red de internet para recibir clases virtuales en tanto que 62 estudiantes, no posee condiciones necesarias para recibir clases virtuales.

\section{Abstract}

Introduction: The present investigation had the purpose of analyzing the influence of virtual teaching in the school desertion of the students of Basic General Education in the "Jerusalem" Highschool from Ambato city. Objective: To analyze the influence of virtual teaching in the school desertion of the students of Basic General Education in the "Jerusalem" Highschool from the Ambato city. Methodology: The methodology used had a cuali-quantitative approach that was focused on an exploratory and descriptive analysis in the selected population. As a technique for collecting the information, it was used a validated survey with a questionnaire of ten objective multiple-choice questions with a sample of 116 students. After collecting data, the proposed hypothesis was evidenced. Result: The results obtained show that a large percentage of students leave school because they have few 
technological tools. This has caused feelings of frustration in students, teachers, and parents, making the teaching-learning process difficult. Conclusions: As a conclusion, it can be determined that of a total of 116 students investigated, only 54 have an internet connection in order to receive virtual classes, in so far as, 62 students do not have the necessary conditions to receive virtual classes.

\section{Introducción}

Según Bocanegra (2020), la clase virtual desde el punto de vista del sistema educativo cobró mayor visibilidad en el transcurso del 2020 debido a la pandemia del covid-19 que formuló nuevos problemas para la educación. En este contexto: la conectividad es el encuentro dinámico entre dos personas que generan sinergia, por decirlo idealmente, aunque en la realidad sea lo contrario. El encuentro dinámico dependerá, principalmente, de las capacidades del docente, porque es el único responsable que está detrás del monitor y graba pequeñas orientaciones en su sala o en cualquier escenario modesto. La conectividad es la sinergia didáctica entre el talento humano del docente y los recursos a disposición, donde uno es protagonista, pero, al mismo tiempo, se subordina al papel de la tecnología. La conectividad es la necesidad que une espacios distantes con el dinamismo del docente para motivar el desarrollo humano desde el hogar.

"Acceder a la conectividad es una pieza fundamental que ojalá se transforme en un recurso irrenunciable en nuestras escuelas dado que hoy ya es evidente que el reparto del abecedario se vehiculiza con lápiz, papel, teclados y bytes". (Ziegler, S., 2020). Una de las barreras a superar para asegurar la continuidad educativa es promover el acceso equitativo a los espacios educativos a través de la conexión a los recursos de Internet y herramientas TIC. A través de la promoción del acceso a las oportunidades digitales, se impulsa la permanencia y culminación de la experiencia educativa. Estas acciones están enfocadas especialmente en niños, niñas y adolescentes refugiados, migrantes y la comunidad de acogida, expuestas al riesgo de abandono por falta de recursos. Organización de las Naciones Unidas para la Educación, la Ciencia y la Cultura (UNESCO, 2021)

Entendiendo a la conectividad como la interacción entre el entorno y las personas a través del acceso a internet o las TIC, se establece una dinámica de transferencia de conocimiento fundamental para el desenvolvimiento de los ambientes educativos. La conectividad permite a docentes y estudiantes compartir, revisar, entender y divulgar la información necesaria para la ejecución de sus clases. Sin embargo, la Comisión Económica para América Latina (CEPAL, 2020), ha planteado que las soluciones de educación en línea ante la situación del covid-19 y la suspensión escolar resultan inviables 
para un $46 \%$ de los niños entre 5 y 12 años de la región que vive en hogares sin conectividad a Internet. Esto representa que más de 32 millones de niños en 13 países de América Latina y el Caribe permanecen excluidos de las chances de recibir educación en línea (Ziegler, S., 2020). Según García y Pérez (2020), la comunicación es el epicentro de la transformación que vivimos y se convierte en aspecto fundamental cuando hablamos de educar a las nuevas generaciones. Por otro lado, la penetración de los dispositivos móviles hace que esta participación alcance cada día mayores nichos poblacionales. La cuestión que emerge ante un panorama de inexorable penetración tecnológica y conectividad es si la alfabetización mediática y digital resulta realmente adecuada para que esa participación resulte crítica y democrática y la educación en comunicación esté siendo efectiva. La multiplicación de pantallas, plataformas y herramientas de comunicación y creación de contenidos dotan a la ciudadanía de infinitas posibilidades de comunicación e interacción, pero también de aprendizaje a través de dispositivos, recursos en abierto y herramientas digitales que amplifican las opciones educativas fuera de las paredes del aula.

La conectividad permite la comunicación con otros y su entorno, el intercambio de datos a través de herramientas como los correos electrónicos, las redes sociales o incluso las plataformas de almacenamiento de información. Las personas cada vez más permanecen conectadas a la tecnología lo que les permite permanecer comunicados con el mundo y los diferentes acontecimientos que se generan. En el campo de la educación, la conectividad es un puntal para la transmisión de saberes pues posibilita la comunicación entre todos los integrantes de un sistema educativo. La vinculación necesaria entre comunicación y educación se torna más patente y necesaria que nunca cuando hacemos alusión a las redes sociales y su influencia sobre todos los ámbitos de nuestra vida. El alcance de las redes como espacios masivos de comunicación e interacción fáciles de utilizar por todas las edades lo convierten en foco de atención (García y Pérez, 2020).

Según Ubilla-Bravo (2017), la accesibilidad es el potencial para alcanzar o conseguir oportunidades que se distribuyen en el espacio, se considera como la facilidad con la que un elemento alcanza a otro. Ortiz (2017), afirma que la accesibilidad en Ecuador debe ser entendida desde una perspectiva amplia, es decir comprender que no se alcanza a cubrir eso por el simple hecho de que las personas puedan acceder a un computador y a una conexión de internet. En ese sentido, se refiere al acceso material de los equipos necesarios para conectarse; posteriormente resalta que parte del acceso debe ser también el interés de las personas en hacerlo y comprender la importancia que aquello tiene en la sociedad del conocimiento; en tercer lugar, reconoce que es fundamental la alfabetización digital y contar con las habilidades necesarias para utilizar adecuadamente las nuevas tecnologías; y, finalmente, se reconoce como fundamental que exista una igualdad de oportunidades en el acceso. 
Universalidad. - Pincay (2021), manifiesta que las personas conectadas pueden interactuar, facilitando los espacios colaborativos muy útiles para los negocios, la educación, la participación en las redes sociales, etc.

Variedad. - Pincay (2021), ofrece la posibilidad de acceder a una gran gama de información mediante la World Wide Web $(W W W)$ y otros servicios como transferencias de ficheros, mensajería, etc.

La deserción escolar. - según estadísticas de la Organización de las Naciones Unidas para la Educación, la Ciencia y la Cultura (UNESCO, 2019), se proyecta que más de 3,1 millones de niños, niñas y adolescentes en América Latina y el Caribe podrían no regresar nunca a la escuela debido al COVID-19. A nivel mundial, es probable que esta región sufra el segundo mayor descenso en la matrícula escolar (1,83 por ciento) entre todas las regiones. Ya el porcentaje de niños que no recibe educación alguna, ni presencial ni de forma remota, se ha disparado del 4 al 18 por ciento en los últimos meses. Los niños, niñas y adolescentes con discapacidad corren un mayor riesgo de abandonar la escuela, junto con las adolescentes que están embarazadas o ya son madres y quienes viven en la pobreza extrema. En general, en los países de América Latina y el Caribe, el 21 por ciento de los niños, niñas y adolescentes de los hogares más pobres no recibe educación de ningún tipo en comparación con el 14 por ciento de los hogares más ricos.

Es esencial conceptualizar el fenómeno de la deserción escolar desde el punto de vista de varios autores de tal modo que se reconozca la diferencia respecto de otros fenómenos educativos. Existen ciertas definiciones relacionadas al futuro y sueños de las personas que desertan de la escuela en las que se relaciona este fenómeno con la consecuencia directa de la falta de oportunidades futuras que se podrían presentar. En tal sentido se consideran a la deserción escolar como: El abandono de parte de los educandos y educadores si nos referimos a las instituciones educativas, no sólo de las aulas donde se adquieren conocimientos, sino también el abandono de sus sueños y perspectivas de una vida futura provechosa y responsable que los llevaría a invalidar su futuro, el cual no es mañana sino hoy.

Velasteguí (2019), analizan el concepto de la deserción escolar desde el punto de vista de los factores que la causan, mencionan que "Se manifiesta que deserción escolar es la renuncia de los estudios por varios factores, como causa principal es el bajo rendimiento, la falta de motivación apoyo de los padres de familia y su acompañamiento en sus clases como la recepción y la entrega de tareas esta debe ser un principal responsabilidad establecidas en el hogar"

Relacionando la deserción escolar como la desvinculación del sistema educativo por parte del estudiante matriculado y por decisión propia se encuentra que según la UNESCO (2020) en su artículo "La formación técnica como respuesta bisagra para reducir la 
potencial deserción causada por el Coronavirus" se define a la deserción escolar como: La deserción se define como el porcentaje de estudiantes que, habiendo estado matriculado en un año, deja de estudiar y no vuelve a matricularse en el siguiente año. En la misma línea de investigación Requena (2019), indican que "el número de alumnos que se han matriculado en su grado correspondiente y por varios motivan no culminan sus estudios o no se matriculan el año siguiente por bajas calificaciones y tienden a buscar trabajado para ayudar en su hogar esta problemática se visualiza en los niveles superiores".

De acuerdo con Suberviola (2021) la deserción escolar presenta un carácter multidimensional, con factores interrelacionados. Se plantea cuatro dimensiones: personal, contexto familiar, social y sistema educativo. De acuerdo con Román (2013), sin lugar a duda los factores socioeconómicos (extraescolares), el nivel socioeconómico de los estudiantes es el principal y reiterado factor externo asociado al abandono y la deserción escolar. En todos los estudios revisados, la pobreza o los bajos ingresos familiares son claros determinantes de este fenómeno. En algunos casos, muy ligados a la necesidad de trabajo por parte del estudiante, en otros poniendo en la balanza los costos de oportunidad real de seguir estudiando en función del beneficio futuro. Así, la probabilidad de abandonar la escuela es significativamente mayor, en los estudiantes pertenecientes a los primeros quintiles de ingreso en todos los países.

Con todo, la vulnerabilidad social y económica, emerge quizás como el determinante o factor externo de mayor relevancia para el abandono y la deserción escolar. Fragilidad que se expresa en términos económicos (sectores de menores ingresos; trabajo, características del entorno), capital cultural de los padres (escolaridad, expectativas, redes) y universo simbólico de los propios estudiantes (proyección y expectativas de concreción de proyectos de vida que incluyen estudios superiores). En esta construcción, aparece como claves la experiencia de deserción en padres y/o hermanos, así como el tener compañeros o amigos desertores o en camino a llegar a ser uno de ellos. Además, según Valdivia et al. (2017), los factores económicos se caracterizan por situaciones financieras tanto de la familia como del mismo estudiante que influyen en el ingreso y permanencia en el sistema educativo. Existen diferentes motivos que propician el abandono escolar, y en específico los motivos relacionados con la situación económica, son: Escasa motivación de los padres de familia y docentes, escasas herramientas tecnológicas, problemas económicos, baja conectividad, escaso interés de docentes y estudiantes, quehaceres del hogar, cuidar a algún familiar, los gastos educativos son elevados, estaba interesado en trabajar. Entre otras razones señalan Lozano y Maldonado (2019), quienes citan a varios autores que "las limitaciones económicas se demuestran cuando el estudiante no logra costearse sus estudios lo que se ve obligado a un abandono de su unidad educativa. Muchas veces no solo es por problemas económicos si no por factores sociales, culturales y tecnológicos. Factores propios del sistema educativo 
(intraescolares), según el estudio realizado por Hernández y Alcaraz (2018), "se han contemplado factores en la dimensión educativa, de los cuales destacan los aspectos asociados a las políticas educativas, pedagógicos, curriculares”. Así, se acrecientan los problemas emanados por la falta de dinero, afectando los años de estudio y perpetuando la condición económica adversa; dañando, una vez más, la competitividad para insertarse en la vida laboral y social del propio individuo. El direccionamiento de los apoyos económicos debe darse a aquellos que realmente lo requieran para continuar sus estudios, pues las zonas con menor prosperidad económica o que se encuentran desfavorecidos y presentan algún grado de vulnerabilidad que inhiben la inversión que genere riqueza, tienen mayor fracaso escolar. Aunque, en el otro extremo, para muchas familias que viven en situación de pobreza las ambiciones educativas son elevadas, pues están conscientes que es una forma vital para salir del trance, lo que las impulsa a esforzarse para no desertar.

Según el estudio realizado por Hernández y Alcaraz (2018), se han contemplado once factores en la dimensión educativa, de los cuales destacan los aspectos asociados a las políticas educativas, pedagógicos y curriculares, la vinculación y cultura escolar, el profesorado y la oferta educativa; frente a la transición entre etapas, la participación familiar en el centro, el impacto de la Educación Infantil, la existencia de la figura del orientador y la disponibilidad de transporte escolar. Los aspectos asociados a las políticas educativas $(53,7 \%)$ condicionan el ámbito escolar, por tanto, la vivencia del alumnado. Los aspectos pedagógicos y curriculares $(48,8 \%)$ están formados por los proyectos educativos y documentos que regulan el centro, la disciplina, atención a la diversidad, práctica escolar reactiva y rigidez del sistema, metodologías y formas de trabajo y la evaluación. En lo que respecta a la vinculación/desvinculación escolar $(31,7 \%)$, el grado de satisfacción/insatisfacción del alumnado hacia el centro escolar depende en gran medida del ambiente percibido en las aulas, de la comunidad de aprendizaje y del sentimiento de pertenencia al centro escolar. Cabe señalar también el "efecto compañero", ya que el grupo de pares incidirá de manera positiva o negativa en la vinculación escolar de cada individuo.

Tipos de deserción. No existen estudios profundos enfocados al análisis del tipo de deserción escolar, sin embargo, Bocanegra (2020) los define así:

Deserción precoz: Cuando un estudiante abandona un programa antes de comenzar habiendo sido aceptado.

Deserción temprana: Cuando se abandona el programa durante los primeros años escolares. 
Deserción temporal: Cuando el alumno hace lo que generalmente se conoce como una baja temporal y cuando se siente seguro regresa al programa educativo para continuar con sus estudios.

Deserción definitiva o total: Cuando el alumno abandona por completo un plan educativo y decide no regresar.

\section{Medidas de prevención}

La división de educación general del Ministerio de Educación del Gobierno de Chile propone a través de una mesa técnica para la prevención de la deserción escolar, las siguientes medidas de prevención.

Generar un relato común sobre las trayectorias escolares positivas y el rol de los adultos responsables. - Los docentes, educadores, asistentes de la educación, directivos y sostenedores están realizando innumerables y valiosos esfuerzos por mantener la cohesión de las comunidades educativas y resguardar las trayectorias de los estudiantes en todo el país, en este período de clases a distancia o remota. Se propone destacar ese esfuerzo dentro de una campaña comunicacional que difunda testimonios de profesores y asistentes, iniciativas impulsadas por directores y sostenedores, y declaraciones de familias que se hayan sentido especialmente acompañadas por la comunidad escolar en este último tiempo. Se propone realizar una campaña de sensibilización a las comunidades educativas con la finalidad de difundir la importancia de analizar las trayectorias escolares de cada estudiante, de manera que estos no queden invisibilizados tras promedios generales. En esta línea, la política comunicacional debe tener como objetivo relevar la importancia de asegurar las trayectorias escolares positivas de todos los niños, niñas y jóvenes del país.

Generar condiciones para que los distintos actores del sistema escolar puedan realizar un trabajo autónomo y activo en promover trayectorias escolares positivas. - La educación a distancia implica un uso intensivo de herramientas virtuales para realizar clases, así como la utilización de medios digitales de comunicación para contactarse con los estudiantes y sus familias. Aunque este cambio afecta a toda la comunidad escolar, son los docentes quienes, por su relación diaria con los alumnos, se ven exigidos con mayor fuerza a emplear estos dispositivos para continuar con la enseñanza. Las vías de comunicación mencionadas requieren de conexión a internet y/o planes de telefonía móvil, en medidas considerablemente mayores a las acostumbradas.

Se propone la entrega de orientaciones para sostenedores y equipos directivos, que expliciten las opciones disponibles para garantizar el financiamiento de la conectividad periódica que requieren los docentes actualmente. Estos lineamientos debieran definir el 
tipo de subvenciones que pueden utilizarse con estos fines, destacando aquellos casos en que se ha flexibilizado temporalmente el uso de recursos estatales.

Fortalecer sistemas de detección oportuna de estudiantes en riesgo de exclusión escolar y apoyar a la comunidad educativa para su uso.- Implementar un Sistema de Alerta Temprana (SAT) que es un instrumento de focalización preventivo que, a partir de información administrativa, identifica el conjunto de condiciones individuales, familiares, del entorno y de los pares de los estudiantes (asistencia, reprobación, nivel socioeconómico, características del entorno social y familiar, entre otros), que tienden a presentarse cuando existe un riesgo de deserción escolar. De esta manera cada director puede identificar a los estudiantes que poseen un alto riesgo de exclusión escolar.

Se propone poner a disposición de todos los establecimientos educacionales una herramienta simple que les permita llevar un registro sistemático del contacto que realizan los docentes y otros profesionales de apoyo con cada estudiante, entregando reportes con información valiosa y orientaciones para directores y sostenedores a partir de la información levantada.

Fortalecer el trabajo en red para enfrentar la multicausalidad de la exclusión escolar, tanto en tiempos de clases a distancia como presenciales. - Las medidas paliativas y de mitigación para disminuir la tasa de contagio del Covid-19 tiene efectos adversos en múltiples dimensiones del bienestar de los niños. La suspensión de las clases presenciales ha sido una de las medidas implementada y ha impactado de forma negativa el proceso de enseñanza y aprendizaje de los estudiantes, así como el vínculo familia-escuela. Es muy probable, por otro lado, que el retorno gradual a la modalidad presencial implique nuevos desafíos, tanto en términos logísticos como en la contención socioemocional y en los procesos pedagógicos.

Distintos equipos educativos han diseñado y piloteado buenas prácticas para contactar y apoyar a sus estudiantes durante el aprendizaje a distancia con el propósito de mantener vivo el vínculo entre las escuelas y cada uno de sus estudiantes. En el proceso de retorno a clases presenciales, seguirán generándose iniciativas que vale la pena destacar y difundir, en la medida que contribuyan a mantener conectados y motivados a los estudiantes.

De esta forma, se propone levantar y diseminar estas buenas prácticas que se están implementando por medio de una plataforma web y otros canales de difusión, en base al material entregado por los propios actores del sistema escolar. El levantamiento debe contemplar el periodo de suspensión de clases presenciales y el retorno gradual a clases presenciales. 
Fortalecer programas existentes de reinserción, reingreso y retención escolar. - Se propone una revisión de los programas de retención, reinserción y reingreso existentes para re focalizar su diseño con el objetivo de mejorar su eficacia y hacerlos más atingentes a las necesidades que presentará el sistema educativo postpandemia. De esa manera, la mesa de expertos propone diferentes cambios a los programas, los cuales deberán ser analizados por el Ministerio de Educación junto a mesas técnicas compuestas por actores claves en función de cada una de las iniciativas y así evaluar en qué medida y con qué profundidad se pueden hacer las mejoras recomendadas.

\section{Metodología}

La investigación es de enfoque cuali - cuantitativo por el hecho que se centra en mediciones objetivas y se realizó un análisis estadístico recopilando los datos mediante encuestas, el método que se aplicó fue el no experimental porque no requiere la modificación de las variables, el diseño fue de campo por que se aplicó una sola vez la encuesta, como instrumento para la obtención de la información se utilizó un cuestionario estructurado y validado. Después de la recopilación de datos, se probó la hipótesis planteada mediante la aplicación del programa Minitab y la medida estadística $\mathrm{X}^{2}$ (Chi cuadrado)

Participantes. - El universo tomado para el desarrollo de la investigación son estudiantes los estudiantes de Educación General Básica de la Unidad Educativa "Jerusalén" del Cantón Ambato, Provincia de Tungurahua, el estudio se realizó con 116 estudiantes. Como resultado, se pudieron obtener datos más confiables durante la encuesta de toda la población.

Instrumento. - La técnica utilizada en el desarrollo del estudio fue la encuesta y como herramienta un cuestionario estructurado con preguntas objetivas.

Protocolo.- Debido a la situación que vive el mundo entero a causa del COVID 19, se mantuvo contacto virtual a través de la plataforma zoom con las autoridades de la institución educativa "Jerusalén" del Cantón Ambato para iniciar la investigación, posteriormente se envía una carta por correo electrónico solicitando autorización para recopilar información de los estudiantes .Como parte del procedimiento de recolección de datos, se obtuvo autorización, luego para realizar la encuesta se envió un enlace donde se formuló el cuestionario en Google Forms, para que los estudiantes lo completaran de manera simple y eficiente. Se envió el enlace del formulario al docente responsable de cada grupo de estudiantes de la institución quien fue el encargado de distribuir el formulario a todos los estudiantes que participan en este estudio, una vez enviado el formulario, las respuestas se registrarán para su posterior análisis e interpretación, siendo el objetivo analizar la información obtenida de los estudiantes. Para el procesamiento estadístico de los datos y resultados de la investigación se utilizó el paquete estadístico 
Minitab, en donde se procedió a ingresar los datos, para el cálculo de $\mathrm{X}^{2}$ (Chi Cuadrado) luego realizar el análisis e interpretación de los datos obtenidos.

\section{Resultados}

Se consideró una encuesta establecida en la metodología que nos permiten mirar objetivamente la realidad referente las clases virtuales y la deserción escolar de los estudiantes de una importante institución educativa del cantón Ambato en la época de pandemia.

Para el acceso a su clase virtual usted lo realiza por medio de:

\section{Tabla 1}

Acceso clases virtuales

\begin{tabular}{lcc}
\hline & Frecuencia & Porcentaje \\
\hline Plan de Internet & 19 & $16 \%$ \\
Datos móviles & 17 & $15 \%$ \\
Internet del representante legal & 16 & $14 \%$ \\
Internet compartido con familiares & 22 & $19 \%$ \\
Internet prestado & 42 & $36 \%$ \\
\hline \multicolumn{1}{c}{ TOTAL } & 116 & $100 \%$ \\
\hline
\end{tabular}

Fuente: Encuestas

\section{Figura 1}

\section{Acceso a las clases virtuales}

Para el acceso a su clase virtual usted lo realiza por medio de:

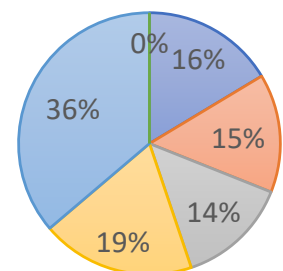
$\square$ Internet propio de casa
$\square$ Datos móviles
$\square$ Internet del trabajo del representante $\square$ Internet compartido con familiares
$\square$ Internet prestado
$\square$ Otros.

Fuente: Datos tabulados de las encuestas

Análisis Descriptivo: De un total de 116 estudiantes que representan el 100\%; 42 estudiantes que representa el $36 \%$ consideran que para el acceso a la clase virtual lo 
realiza por medio de un internet prestado; 22 estudiantes que representa el $19 \%$ que cuentan con un internet compartido con familiares; 19 estudiantes que representa el 16\% con un internet propio en casa; 17 estudiantes que representa el $15 \%$ con datos móviles y 16 estudiantes que representa el 14\% donde cuenta con internet del trabajo del representante para la clase virtual, los resultados obtenidos se puede evidenciar que en la mayoría de los dicentes cuentan con un internet prestado para recibir las clases virtuales, por consecuente no logran ingresar todos los días a las clases de una manera adecuada, dificultándoles su formación académica.

Con qué frecuencia ingresa a la clase virtual

Tabla 2

Frecuencia ingreso a la clase virtual

\begin{tabular}{lcc}
\hline Ingreso a la clase virtual & Frecuencia & Porcentaje \\
\hline Todos los días & 15 & $13 \%$ \\
Tres días a la semana & 22 & $19 \%$ \\
Dos días a la semana & 38 & $33 \%$ \\
Un día a la semana & 23 & $20 \%$ \\
No ingreso a la clase virtual. & 18 & $16 \%$ \\
\hline TOTAL & 116 & $100 \%$ \\
\hline
\end{tabular}

Fuente: Encuestas

\section{Figura 2}

Con qué frecuencia ingresa a la clase virtual

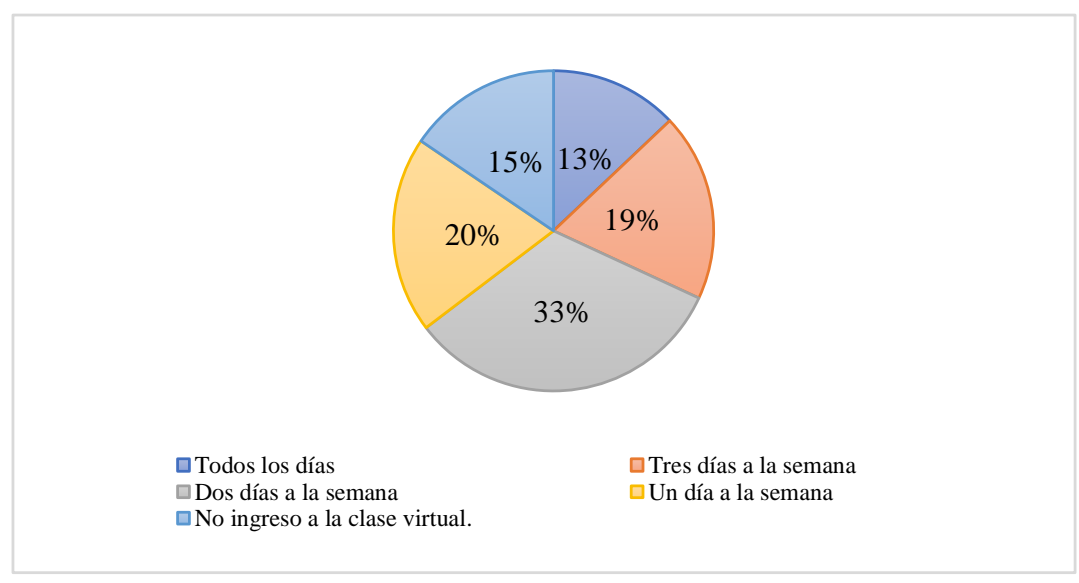

Fuente: Datos tabulados de las encuestas 
Análisis descriptivo: De un total de 116 estudiantes que representan el 100\%; 38 estudiantes que representa el 33\% considera que ingresa a la clase virtual dos días a la semana; 23 discentes que representa el $20 \%$ manifiesta que ingresa un día a la semana; 22 estudiantes que representa 19\% ingresan tres días a la semana; 18 estudiantes que representa el 16\% no ingresan a las clases. Además, 15 estudiantes que representa el 13\% ingresan todos los días de la semana a las clases virtual, prácticamente la asistencia a clases de los estudiantes un grupo de 15 estudiantes asisten con regularidad todos los días, en cambio 83 estudiante marcan irregularidad en su asistencia y 18 estudiante no asisten y cumplen actividades de a través de otros medios como el WhatsApp, correspondiendo a los padres o familiares más cercanos cumplir con el rol de maestros que podría ocasionar el retiro de los estudios, demostrando que estamos preparados para la educación virtual .

\section{Prueba de chi - cuadrado}

Para la prueba de chi cuadrado, se consideró las preguntas, ¿Con qué frecuencia ingresa a la clase virtual? y Para el acceso a su clase virtual usted lo realiza por medio de Usted a las clases virtuales lo realiza mediante: Plan de internet, datos móviles, internet del representante legal, Internet compartido con familiares, Internet prestado

\section{Tabla 3}

Frecuencia de acceso a clases a través del internet

\begin{tabular}{|c|c|c|c|c|c|c|}
\hline $\begin{array}{c}\text { Con qué frecuencia } \\
\text { ingresa a la clase } \\
\text { virtual }\end{array}$ & $\begin{array}{c}\text { Todos los } \\
\text { días }\end{array}$ & $\begin{array}{c}\text { Tres días } \\
\text { a la } \\
\text { semana }\end{array}$ & $\begin{array}{c}\text { Dos días a la } \\
\text { semana }\end{array}$ & $\begin{array}{c}\text { Un día a la } \\
\text { semana }\end{array}$ & $\begin{array}{l}\text { No } \\
\text { ingreso a } \\
\text { la clase } \\
\text { virtual. }\end{array}$ & Total \\
\hline Total respuesta & 15 & 22 & 38 & 23 & 18 & 116 \\
\hline $\begin{array}{l}\text { Para el acceso a su } \\
\text { clase virtual usted lo } \\
\text { realiza por medio } \\
\text { de: }\end{array}$ & $\begin{array}{l}\text { Plan de } \\
\text { Internet }\end{array}$ & $\begin{array}{l}\text { Datos } \\
\text { móviles }\end{array}$ & $\begin{array}{l}\text { Internet del } \\
\text { representante } \\
\text { legal }\end{array}$ & $\begin{array}{c}\text { Internet } \\
\text { compartido } \\
\text { con } \\
\text { familiares }\end{array}$ & $\begin{array}{l}\text { Internet } \\
\text { prestado }\end{array}$ & Total \\
\hline Total respuesta & 19 & 17 & 16 & 22 & 42 & 116 \\
\hline Total & 34 & 39 & 54 & 45 & 60 & 232 \\
\hline
\end{tabular}

Fuente: Encuestas 


\section{Tabla 4}

Frecuencias observadas y esperadas

\begin{tabular}{ccccccc}
\hline V.I & 15 & 22 & 38 & 23 & 18 & 116 \\
& 17,00 & 19,50 & 27,00 & 22,50 & 30,00 & \\
\hline V.D & 19 & 17 & 16 & 22 & 42 & 116 \\
& 17,00 & 19,50 & 27,00 & 22,50 & 30,00 & \\
\hline Todo & 34 & 39 & 54 & 45 & 60 & 232
\end{tabular}

Fuente: Encuestas

Con tres grados de libertad, un nivel de confiabilidad de $95 \%$, y con una significancia de $\alpha=0,05$. Se obtiene en la tabla el valor de Chi cuadrado tabular de $X t 2=9.48$, mientras que el valor de Chi cuadrado calculado de $X c 2=19,69$, entonces con los valores obtenidos se rechaza la hipótesis nula y se acepta la hipótesis alterna que expresa que dice: La educación virtual incide en la deserción escolar en la pandemia 2020 de los estudiantes de Educación General Básica de la Unidad Educativa “Jerusalén” del Cantón Ambato.

\section{Figura 3}

Acceso a las clases virtuales

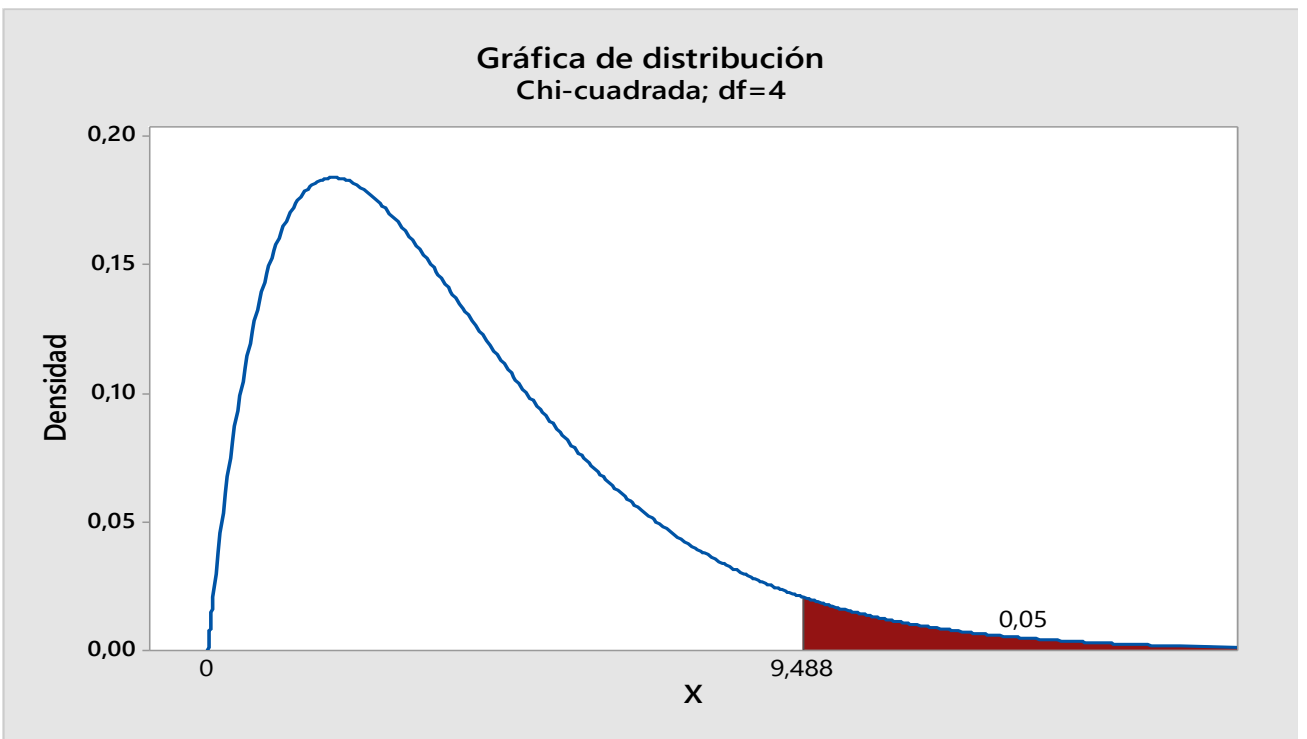

Fuente: Datos tabulados de las encuestas 


\section{Conclusiones}

- La enseñanza virtual en la deserción escolar de los estudiantes de Educación General Básica de la Unidad Educativa "Jerusalén" del Cantón Ambato, ha permitido identificar que los estudiantes tienen limitaciones para acceder a las clases virtuales porque el $83 \%$ de los encuestados no tiene las condiciones que permitan recibir las clases virtuales incluso de este grupo 42 estudiantes utilizan internet prestado, viéndose perjudicados con la asistencia donde solamente el $13 \%$ acude todos los días y 93 estudiante acuden irregularmente en un rango de 3 a 1 día, en cambio 18 estudiantes no ingresan a clases deduciendo que la responsabilidad de la educación estaría a cargo de los familiares más cercanos del alumno.

- Las condiciones económicas en la mayoría de los padres de familia por la falta de trabajo han limitado que sus hijos dispongan de los recursos tecnológicos necesarios, del acceso al internet y consecuentemente su asistencia no es regular a la escuela.

- La institución educativa debe adoptar estrategias que permitan cumplir con los objetivos educativos planificados para el año lectivo en función de las necesidades de los estudiantes, sus condiciones de contexto socioeconómico y tecnológico, porque al no asistir el estudiante a clases se contribuye a aumentar los niveles de analfabetismo en el país.

- La deserción escolar en la época de pandemia Covid-19 ha sido determinante y ha marcado cambios en la sociedad y en el aspecto educativo ha dejado huellas de carácter psicológico y de aprendizaje que se verán reflejadas en grandes limitaciones de acceso a la tecnología como consecuencia del ausentismo escolar de quienes no han podido asistir regularmente a la escuela.

\section{Referencias bibliográficas}

Bocanegra, Vilcamango (2020). La conectividad: Necesidades y políticas educativas. Revista de Investigación En Gestión Industrial, Ambiental, Seguridad y Salud En El Trabajo-GISST, 2(2), 61-75.

CEPAL. (2020). Universalizar el acceso a las tecnologías digitales para enfrentar los efectos del COVID-19 (CEPAL (ed.)).

García y Pérez (2020). Comunicación y Educación en un mundo digital y conectado. Presentación. Icono14, 18(2), 1-15. https://doi.org/10.7195/RI14.V18I2.1580

Hernández y Alcaraz (2018). Factores incidentes en el abandono escolar prematuro. Revista de Investigación En Educación, 16(2), 182-195. 
Lozano Treviño, D. F., \& Maldonado Maldonado, L. (2020). Asociación entre factores económicos y sociales con la propensión de deserción escolar en colegios militarizados. Revista de Estudios y Experiencias En Educación, 19(40), 35-52. https://doi.org/10.21703/rexe.20201940lozano2

Ortiz Ruiz, Y. (2017). Accesibilidad usando las tecnologías de la información y la comunicación. Conocimiento Educativo, 4, 25. https://doi.org/10.5377/ce.v4i0.5650

Pincay Romero, K. G. (2021). Características de la conectividad a Internet en el cantón Pasaje. Universidad y Sociedad, 4(1), 6.

Requena Mendizabal, C. A. (2019). Relación entre los factores sociales, económicos, culturales y el abandono de la escuela en los alumnos de educación secundaria, UGEL 02 RÍMAC, Año 2018. Universidad de San Martin de Porres.

Román Carrasco, M. (2013). Factores Asociados al Abandono y la Deserción Escolar en América Latina: Una Mirada en Conjunto. Revista Iberoamericana Sobre Calidad, Eficacia y Cambio En La Educación, 11(2), 33-59. https://doi.org/10.1016/00415553(78)90043-5

Suberviola, I. (2021). Análisis de los factores predictivos del abandono escolar temprano. Vivat Academia, 25-52. https://doi.org/10.15178/va.2021.154.e1373

Ubilla-Bravo, G. (2017). Accesibilidad y conectividad geográfica en áreas rurales. Caso de la comuna de María Pinto, Chile. Papeles de Geografía, 63(63), 195. https://doi.org/10.6018/geografia/2017/299271

UNESCO. (2019). Indicadores de la UNESCO sobre la universalidad de Internet: marco para la evaluación del desarrollo de Internet (UNESCO (ed.)).

UNESCO. (2020). La formación técnica como respuesta bisagra para reducir la potencial deserción causada por el Coronavirus. https://es.unesco.org/news/formacion-tecnica-como-respuesta-bisagra-reducirpotencial-desercion-causada-coronavirus

UNESCO. (2021). Continuidad educativa en Ecuador: entrega de kits de conectividad. https://es.unesco.org/news/continuidad-educativa-en-ecuador-entrega-de-kits-deconectividad

Valdivia Gutiérrez, M. de los Á., \& Barba Barrera, A. (2017). La escasez de recursos económicos como causa de deserción. Revista: Atlante. Cuadernos de Educación y Desarrollo.

Velasteguí López, E. (2019). Las pizarras digitales y su impacto didáctico en la educación 


\section{DDigital}

\section{ISSN: 2600-5859}

Vol. 6 No. 1.4, pp. 995 - 1012 , marzo 2023

www.concienciadigital.org

superior.

Explorador

Digital,

$3(1)$,

49-63.

https://doi.org/10.33262/exploradordigital.v3i1.346

Ziegler, S. (2020). La conectividad: un imperativo en la agenda educativa regional. Flacso. https://www.ecys.flacso.org.ar/post/la-conectividad-un-imperativo-en-laagenda-educativa-regiona

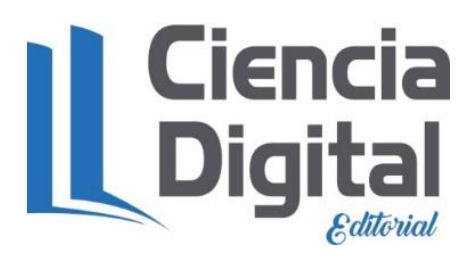


El artículo que se publica es de exclusiva responsabilidad de los autores y no necesariamente reflejan el pensamiento de la Revista Conciencia Digital.

\section{Ciencia
Digital
Ethtoul}

El artículo queda en propiedad de la revista y, por tanto, su publicación parcial y/o total en otro medio tiene que ser autorizado por el director de la Revista Conciencia Digital.
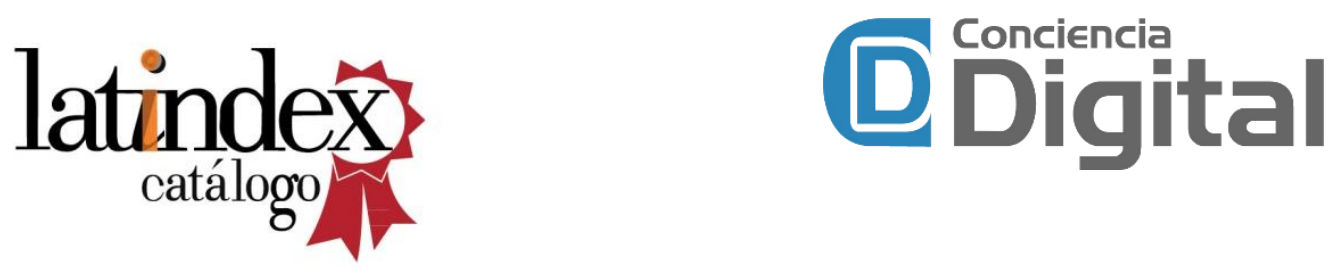

Indexaciones

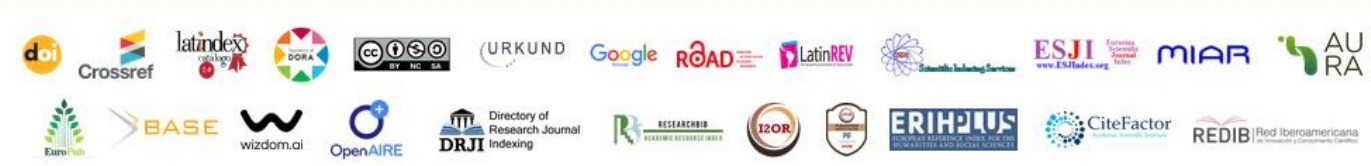

